\title{
Adalimumab como alternativa terapéutica en el síndrome de Vogt Koyanagi Harada refractario al infliximab: reporte de caso
}

\author{
Adalimumab, therapeutic alternative in the Vogt Koyanagi Harada syndrome refractory to
} infliximab: case report

\author{
Eugenio Franklin Moya Ayre ${ }^{1}$, Iván Bermúdez Maldonado² \\ 'Médico residente de Oftalmología, Hospital Nacional Guillermo Almenara Irigoyen, Lima, Perú. \\ ${ }^{2}$ Médico Oftalmólogo, servicio de úvea, Hospital Nacional Guillermo Almenara Irigoyen, Lima, Perú.
}

\begin{abstract}
Resumen
El sindrome de Vogt Koyanagi Harada (VKH) consiste en una panuveitis bilateral que forma parte de los síndromes uveomeníngeos. El tratamiento en estadio crónico es difícil por presentar pobre respuesta a la inmunomodulación, por lo que se recurre a opciones terapéuticas como agentes biológicos tipo anti-TNF alfa. Se describe el caso de una paciente con VKH severo y resistencia al infliximab, quien mostró respuesta al adalimumab. El adalimumab es un anticuerpo monoclonal humanizado efectivo en casos de resistencia al infliximab en pacientes con síndrome de VKH crónico persistente. El caso es de interés por ser infrecuente la resistencia a este medicamento en la práctica clínica, y el uso del activador de plasminógeno tisular contribuyó significativamente en la mejoría visual.

Palabras clave. Sindrome Uveomeningoencefalitico, Vogt Koyanagi Harada; Adalimumab, Infliximab.
\end{abstract}

\section{Abstract}

Vogt Koyanagi Harada syndrome (VKH) is a bilateral panuveitis included in the uveomeningeal syndromes. Treatment of its chronic stage is difficult because of poor response to immunomodulation. Other therapeutic options include biological agents such as antiTNF alpha. We present the case of a patient with severe VKH resistant to infliximab that responded to adalimumab. Adalimumab is a humanized monoclonal antibody effective when there is resistance to infliximab in patients with chronic persistent VKH syndrome. The case presented is interesting because of uncommon resistance to this drug in the clinical practice; the use of tissue plasminogen activator contributed significantly to visual improvement.

Keywords. Uveomeningoencephalitic syndrome, Vogt Koyanagi Harada; Adalimumab, Infliximab.

An Fac med. 2016;77(1):55-8 / http://dx.doi.org/10.15381/anales.v77i1.11554

\section{INTRODUCCIÓN}

El síndrome de Vogt Koyanagi Harada (VKH) corresponde a una enfermedad autoinmune multisistémica que se manifiesta por reacción inflamatoria que afecta los órganos pigmentados, especialmente la úvea y el pigmento retinal, y caracterizado por panuveitis granulomatosa bilateral. A su vez, altera de forma variable los pares craneales II y VIII (hipoacusia neurosensorial uni o bilateral), meninges, encéfalo, piel y anexos (vitíligo, poliosis, alopecía y calvicie.) Los pacientes pueden presentar complicaciones oculares graves, como la reducción de la agudeza visual y una posible ceguera ${ }^{(1-3)}$.

Los corticosteroides en dosis altas son la piedra angular para evitar complicaciones y la progresión a la fase crónica recurrente de la enfermedad. Los fármacos inmunosupresores se suelen añadir en el caso de falla o intolerancia a los corticosteroides. Sin embargo, en algunos pacientes, el síndrome de VKH permanece activo o presenta recidivas, conllevando a daño ocular permanente ${ }^{(4)}$.

Hay estudios que describen la utilidad del infliximab, un anticuerpo monoclonal IG1 quimérico murino y humano anti TNF-alfa usado en pacientes con $\mathrm{VKH}$ refractario. El adalimumab es un anticuerpo monoclonal IG1 completamente humanizado, anti-TNFalfa, que puede ser utilizado para casos refractarios. Existe poca información disponible al momento acerca de este tratamiento en el caso de resistencia al infliximab en el síndrome de VKH ${ }^{(5)}$.

El objetivo del presente estudio es dar a conocer sobre la eficacia y ventajas de la nueva terapia anti-TNF, el adalimumab, en una paciente resisten- te al infliximab, sobre el que todavía no hay muchos estudios que describan su eficacia en este síndrome de VKH refractario.

\section{CASO CLÍNICO}

Presentamos el caso de una mujer procedente del norte del país, de 24 años de edad, con antecedente de cirugía refractiva por excimer láser en ojo derecho por miopía alta de 10DP. Sufría de enfermedad de Vogt Koyanagi Harada de 4 años de evolución, con otalgia e hipoacusia asociada a desprendimiento de retina seroso bilateral, poliosis (figura 1) y vitíligo, siendo catalogada como síndrome de VKH. En la retinografía y angiografía se observó aspecto de sunset glow fundus (despigmentación de la coroides) y múltiples áreas de fuga de morfología puntiforme (figuras 2 y 3 ). 


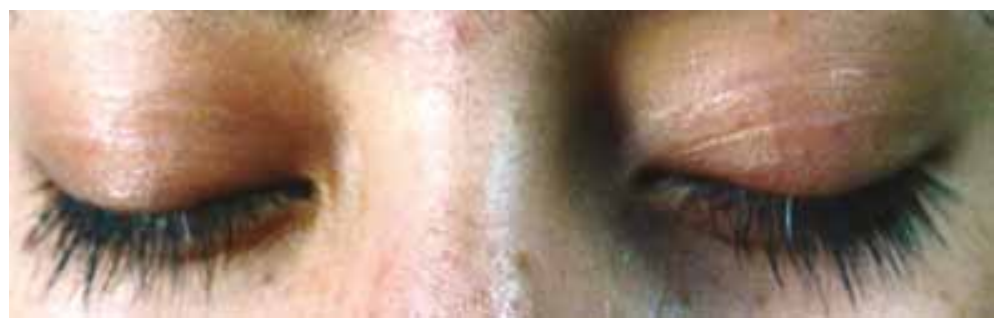

Figura 1. Poliosis.

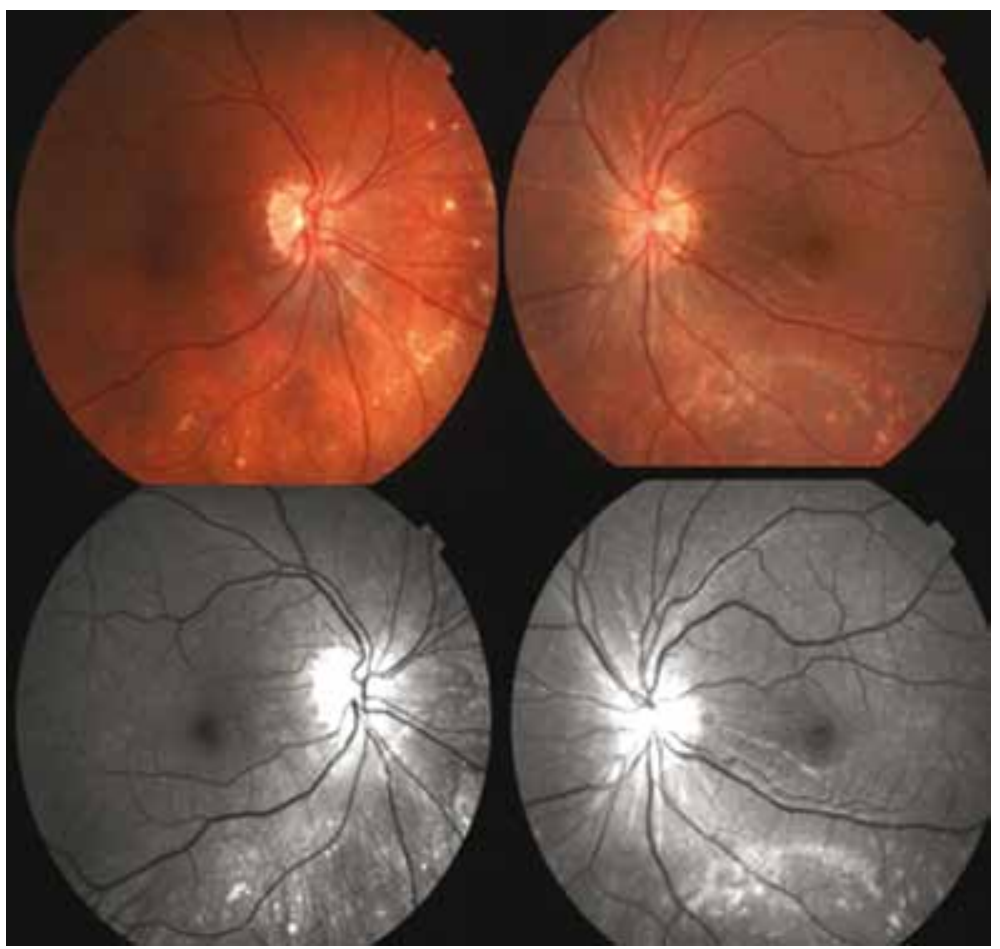

Figura 2. Retinografía. Aspecto de sunset glow fundus.

Recibió prednisona $1 \mathrm{mg} / \mathrm{kg}$, disminuyendo progresivamente la dosis, y azatioprina $150 \mathrm{mg} /$ día por 7 meses más triamcinolona subtenoniana, con aparente buen control. Al término de este plazo tuvo recaída, aplicándose entonces un pulso de metilprednisolona 250 $\mathrm{mg} / 6$ horas/3 días; se cambió la medicación e inició ciclosporina 200 mg/día más prednisona $1 \mathrm{mg} / \mathrm{kg}$, reduciendo progresivamente la dosis. Hubo mejoría aparente por unos 6 meses y luego presentó nueva recaída.

Posterior a ello y por no haber tenido buena respuesta a la ciclosporina, se inició pulsos mensuales de ciclofosfami- intraocular más elevación de la presión intraocular (PIO).

Después de evaluar el caso, se decidió cambiar de medicación a adalimumab subcutánea quincenal, recibiendo 4 dosis al momento de su ingreso a nuestro hospital, con buena respuesta terapéutica.

La paciente presentó facies cushinoide característica por uso crónico de corticoides a altas dosis, además aumento de la $\mathrm{PIO}$ en ambos ojos ( $>30)$, posiblemente por el uso crónico de corticoides y/o progresión del cuadro uveítico de fondo. Esto motivó iridectomía periférica en el ojo derecho y, en el ojo izquierdo, vitrectomía por vitreitis moderada más limpieza de lente e iridectomía quirúrgica. La paciente tuvo membrana inflamatoria pupilar posquirúrgica en el ojo izquierdo. Con el uso del activador del plasminógeno tisular intracameral se notó mejoría evidente a las 24 horas de su administración, mejorando la agudeza visual en el ojo izquierdo de movimiento de manos 50 $\mathrm{cm}$ a 20/70 (figura 4).

\section{DISCUSIÓN}

El uso de medicamentos biológicos en las uveítis refractarias así como también el tratamiento inmunosupresor representan gran ayuda para controlar la enfermedad. Hay muy pocos casos descritos del uso de adalimumab en pacientes resistentes al infliximab, particularmente en el síndrome de Vogt Koyanagi Harada refractario, que habitualmente responde favorablemente al infliximab ${ }^{(4,6)}$.

Es importante distinguir inmunosupresión de inmunomodulación para comprender dónde actúan los medicamentos como el infliximab, adalimumab, entre otros, usados con frecuencia en la práctica clínica oftalmológica. Un medicamento inmunosupresor se define en farmacología como aquel capaz de disminuir la respuesta inmune de forma global. Mientras que un inmunomodulador es un fármaco inmunosupresor, pero de acción selectiva, bloqueando 


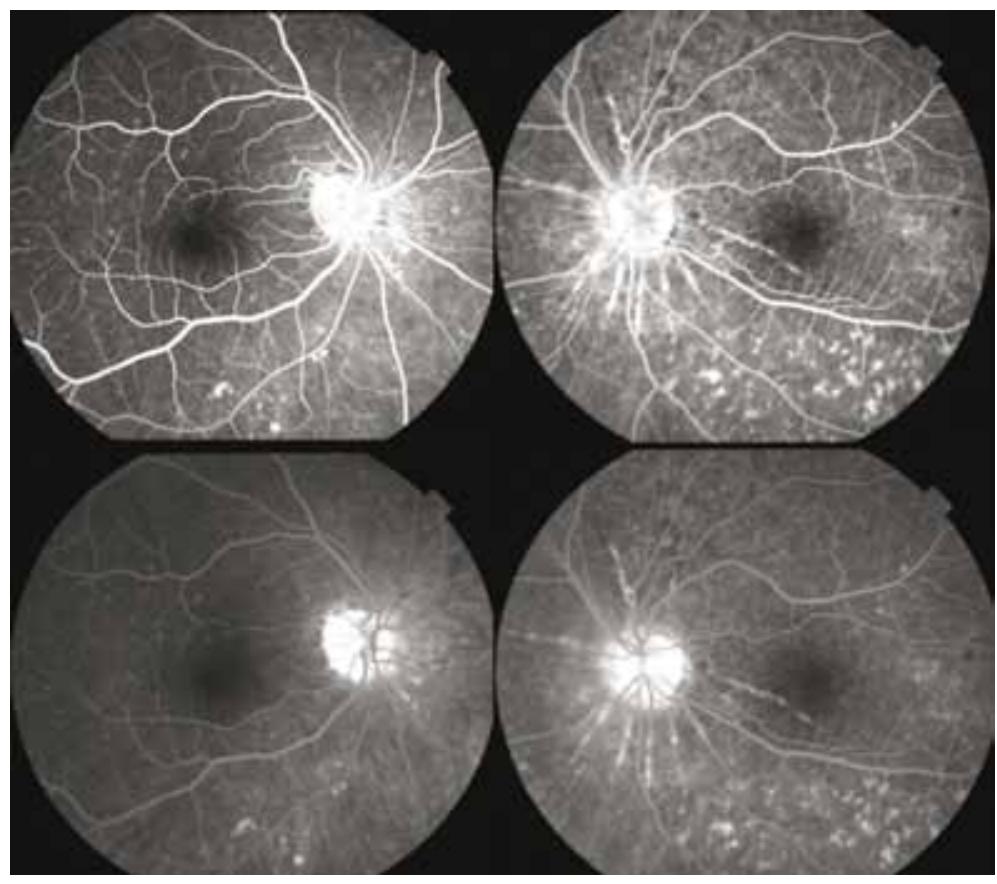

Figura 3. Angiografía. Se aprecia múltiples áreas de fuga de aspecto puntiforme.

con preferencia los mecanismos implicados en el proceso de rechazo.

El factor de necrosis tumoral alfa (FNT $\alpha$ ) es un mediador de la inflamación que modula la respuesta inmunitaria celular. Los datos experimentales muestran que el FNT $\alpha$ es esencial para la eliminación de infecciones intracelulares. La experiencia clínica demues- tra que algunos pacientes tratados con medicamentos anti-FNT $\alpha$, como el infliximab o adalimumab, comprometen sus defensas frente a la infección. Por ello es muy importante una evaluación exhaustiva antes de indicar este medicamento, pues se ha descrito reactivación de hepatitis $\mathrm{B}$, tuberculosis y otras ${ }^{(7,8)}$.
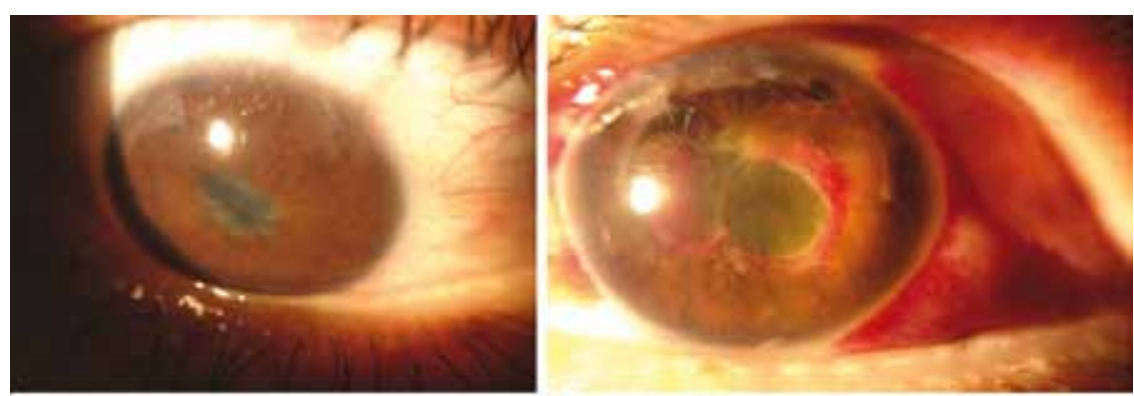

Figura 4. Ojo derecho y ojo izquierdo con membrana pupilar antes y después del tratamiento con activador de plasminógeno tisular en el ojo izquierdo.

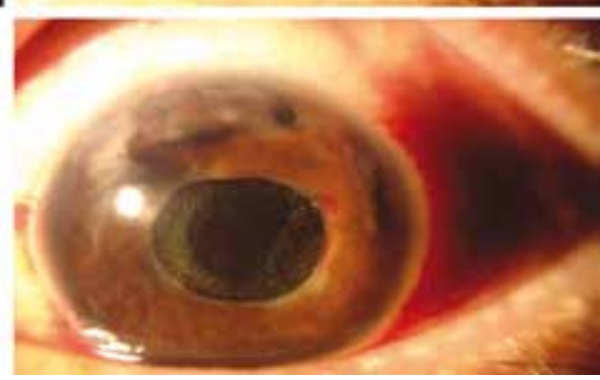

Se pueden desarrollar anticuerpos al infliximab, que han sido asociados con aumento en la frecuencia de reacciones a la perfusión. También se ha observado una asociación entre el desarrollo de anticuerpos frente a infliximab y reducción de la duración de la respuesta. Como habría sucedido en el caso reportado, la administración concomitante de inmunomoduladores se asoció con una menor incidencia de anticuerpos frente a infliximab y reducción en la frecuencia de la perfusión, que debiera ser de dos horas, aproximadamente.

Una ventaja del adalimumab es que se administra por vía subcutánea, lo que permite su administración domiciliaria y proporciona niveles plasmáticos más continuos, mientras que el infliximab precisa la administración intravenosa en medio hospitalario ${ }^{(7)}$.

La evidencia es favorable para los antagonistas TNF-alfa en el tratamiento de las uveítis y enfermedades asociadas. Si bien la aplicación de esteroides peribulbar puede ser benéfica en pacientes con enfermedad leve, las formas severas de la enfermedad frecuentemente requieren esteroides sistémicos con o sin drogas inmunosupresoras, como metotrexato, ciclosporina A o ciclofosfamida. Es decir, el tratamiento de la uveítis crónica o recurrente, por su gravedad, requiere un abordaje similar al utilizado para el control de la inflamación sistémica; es decir, inmunosupresión agresiva. Este régimen debe ir dirigido, idealmente, a controlar los pasos claves en el proceso inflamatorio, controlándolo de manera eficiente y evitando efectos adversos. Sin embargo, hasta 30\% de los pacientes son refractarios a estos regímenes inmunosupresores y cerca de $40 \%$ pueden experimentar recaída durante el curso de la enfermedad, presentando daño ocular irreversible. A finales de los años noventa se introdujeron los antagonistas del TNF en el tratamiento de la artritis reumatoide (AR) y de la enfermedad de Crohn (EC). Estos medicamentos incluyen los anticuerpos monoclonales anti-TNF $\alpha$ infliximab (quimérico) y adalimumab (humaniza- 
do) y el receptor soluble de TNF $\alpha$ etanercept. Ninguno de los inhibidores del TNF $\alpha$ ha sido aprobado para el uso en uveítis por la FDA, excepto en Japón, donde las agencias reguladoras tienen aprobado el uso de infliximab para el tratamiento de la uveítis refractaria ${ }^{(9)}$.

Según una revisión sistemática sobre el uso de adalimumab en enfermedades autoinmunes, que incluye 54 casos, la mayoría de los pacientes era refractaria al tratamiento convencional. De ellos, cuatro tenían síndrome de $\mathrm{VKH}$; uno era resistente al infliximab, uno resistente al daclizumab y los otros dos resistentes a los inmunosupresores convencionales. Este fármaco ha demostrado ser eficaz y seguro, como se podrá observar en el paciente reportado. Sin embargo, uno de los cuatro pacientes de ese estudio fue resistente al adalimumab ${ }^{(7)}$.

Dolz Marco reporta el caso de una paciente con diagnóstico de VKH refractaria al tratamiento con adalimumab y buena respuesta a rituximab de $1 \mathrm{~g}$ en 4 dosis, a 0, 1, 6 y 16 meses. Sin embargo, estos medicamentos no han sido aprobados para tal fin. Pero, parece ser un arma terapéutica importante en casos refractarios ${ }^{(6)}$.

En resumen, el adalimumab en enfermedades autoinmunes en pacientes refractarios al tratamiento convencional ha mostrado ser un fármaco eficaz y seguro, con una mayor experiencia acumulada en pacientes con uveítis y enfermedad de Behcet. El adalimumab parece tener un perfil de eficacia similar al infliximab en las patologías en las que se ha empleado hasta el momento. En cambio, etanercept parece tener menor eficacia en el tratamiento de las uveítis y la sarcoidosis. En este momento, y a la espera de ensayos clínicos que confirmen los resultados previos, la evidencia científica actual no permite recomendar el empleo de adalimumab como primera opción en el tratamiento de las enfermedades autoinmunes sistémicas. El escenario clínico para el empleo del adalimumab queda restringido a aquellos casos refractarios al tratamiento convencional con glucocorticoides e inmunosupresores, y en aquellos pacientes en los que han fracasado otros anti-TNF, bien por ineficacia o por desarrollo de eventos adversos.

Por último, el uso de un activador de plasminógeno tisular recombinante (alteplase) intracameral, a la dosis de 25 ug/0,1 mL, usado en nuestra paciente en una sola aplicación, resulta eficaz en la membrana pupilar inflamatoria posquirúrgica, como demuestran los estudios al respecto ${ }^{(10,11)}$.

\section{REFERENCIAS BIBLIOGRÁFICAS}

1. Lozano Elizondo D, Gutiérrez Alvarado RA. Resonancia magnética en el sindrome de VogtKoyanagi-Harada (uveo-meningoencefalitis). Rev Mex Oftalmol. 1999;73(5):220-6.

2. Damico FM, Bezerra FT, Silva GC, Gasparin F, Yamamoto JH. New insights into Vogt-Koyanagi-Harada disease. Arq Bras Oftalmol. 2009;72(3):413-20.

3. Rodriguez Rivera V, Perez H, Gallego R. Sensori- neural hearing loss evolution in Vogt-KoyanagiHarada syndrome. Acta Otorrinolaringol Esp. 2011;62(6):465-8.

4. Niccoli L, Nannini C, Cassará E, Gini G, Lenzetti I, Cantini F. Efficacy of infliximab therapy in two patients with refractory Vogt-Koyanagi-Harada disease. Br J Ophthalmol; 2009; 93(11):1553-4.

5. Diaz Llopis M, Amselem L, Romero FJ, GarciaDelpech S, Hernández ML. Tratamiento con Adalimumab -nuevo antiTNF- del sindrome de Vogt-Koyanagi-Harada / Adalimumab therapy for Vogt-Koyanagi-Harada syndrome. Arch Soc Esp Oftalmol. 2007; 82(3):131-2.

6. Dolz-Marco R, Gallego-Pinazo R, Diaz-Llopis M. Rituximab in refractory Vogt-Koyanagi-Harada disease. J Ophthal Inflamm Infect. 2011;1:177-80.

7. Diaz-Lagares C, Belenguer R, Ramos Casals M. Revisión sistemática del uso de adalimumab en enfermedades autoinmunes. Eficacia y seguridad en 54 pacientes. Reumatol Clin. 2010;6(3):121-7.

8. Pasadhika S, Suhler EB, Cunningham ET. Biologic therapy for posterior uveitis and panuveitis. Retina Today. 2012 Apr;74-9.

9. Velásquez CJ, Monsalve P, Salazar GI, Rivera A, Zuluaga L, Duran C, et al. Uveitis and anti-TNF therapy. Rev Colomb Reumatol. 2011;18(1):42-54.

10. Dotan A, Kaiserman I, Kremer I, Ehrlich R, Bahar I. Intracameral recombinant tissue plasminogen activator ( $r$-tPA) for refractory toxic anterior segment syndrome. Br J Ophthalmol. 2014 ;98(2):252-5.

11. Tripathi RC, Tripathi BJ. Tissue plasminogen activator therapy for the eye. Br J Ophthalmol. 2005 Nov;89(11):1390-1.

Artículo recibido el 22 de septiembre de 2015 y aceptado para publicación el 27 de diciembre de 2015.

Financiamiento: Trabajo autofinanciado.

Conflicto de intereses: No existen.

Correspondencia:

Eugenio Franklin Moya Ayre

Dirección: Av. Grau 800, La Victoria, Lima

Teléfono: 968182313.

Correo electrónico: moyapharma@hotmail.com 